\title{
A Giant Retroperitoneal Abscess Mimicking Incarcerated Inguinal Hernia
}

Inkarsere Inguinal Herniyi Taklit Eden Dev Boyutlarda Retroperitoneal Abse

\author{
Naciye Sinem Gezer1, Nuri Karabay1, Özkan Alataş1, Ali Çaylak1, Abdullah Taylan1, Koray Atila2 \\ 'Dokuz Eylül University Faculty of Medicine, Department of Radiology, Izmir, Turkey \\ 2Dokuz Eylül University Faculty of Medicine, Department of General Surgery, Izmir, Turkey
}

\section{Introduction}

An 82-year-old man was admitted to the emergency room with an acute left-sided groin pain and scrotal swelling. He has suffered from a groin hernia for two years. Abdominal x-ray demonstrated air-fluid levels in the left upper quadrant suggesting an intestinal obstruction (Figure 1). Abdominal computed tomography (CT)

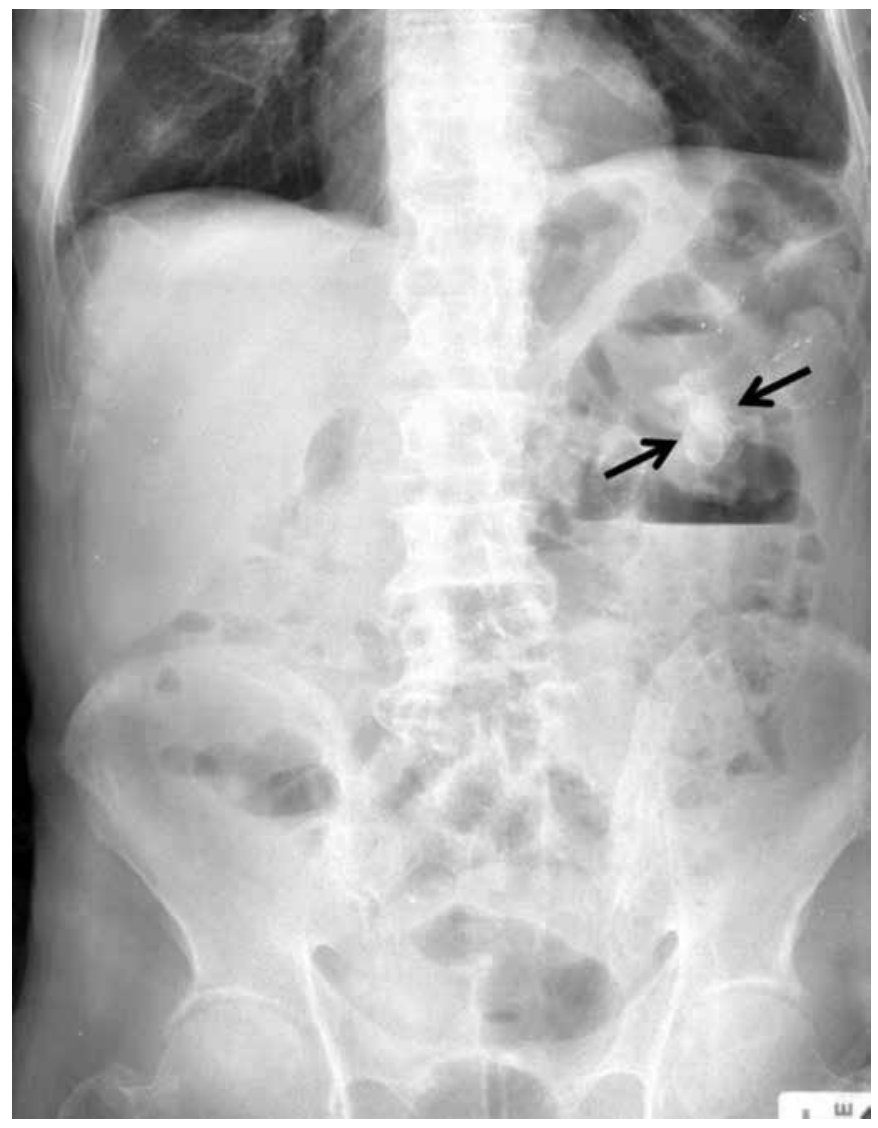

Figure 1. Abdominal x-ray showing staghorn calculi (arrows) in the left kidney and air-fluid levels in the left upper quadrant scan was obtained with an initial diagnosis of an incarcerated inguinal hernia. However, it showed multiple perirenal abscesses and a giant-sized retroperitoneal abscess extending from the retroperitoneal space into the scrotum through the inguinal canal (Figure 2 and 3).

Retroperitoneal abscesses are most frequently seen in the $3^{\text {rd }}$ to 6 th decades of life (1). Gram-negative bacteria, most commonly E. coli, are the cause of infection which usually develops secondary to pyelonephritis, urinary stasis or immune suppression. The onset of clinical manifestations of the infection, including flank, abdomen and groin pain, chills, fever, tachycardia, weakness and anorexia are often insidious $(2,3)$. Laboratory findings include leukocytosis, increased serum creatinine levels and pyuria. The literature emphasizes the possibility of diagnostic delay and postponed treatment of retroperitoneal abscess due to the fact that its prodrome phase may be long. In order to reduce the morbidity and

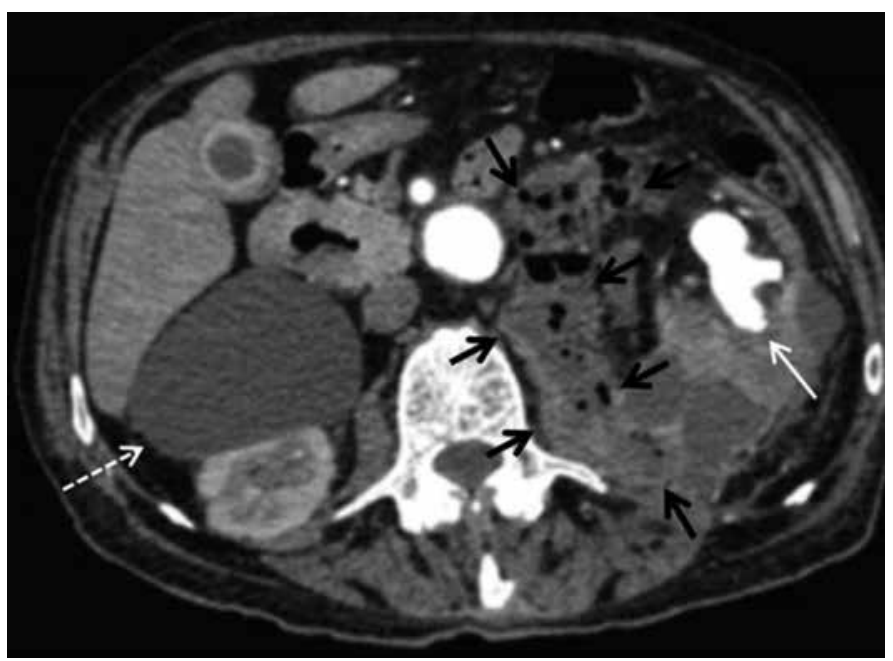

Figure 2. Axial computed tomography image of the abdomen shows staghorn calculi (white arrow) in the left kidney, multiple perirenal abscesses and retroperitoneal abscess containing air. A simple cyst is also detected in the right kidney (dashed arrow). 


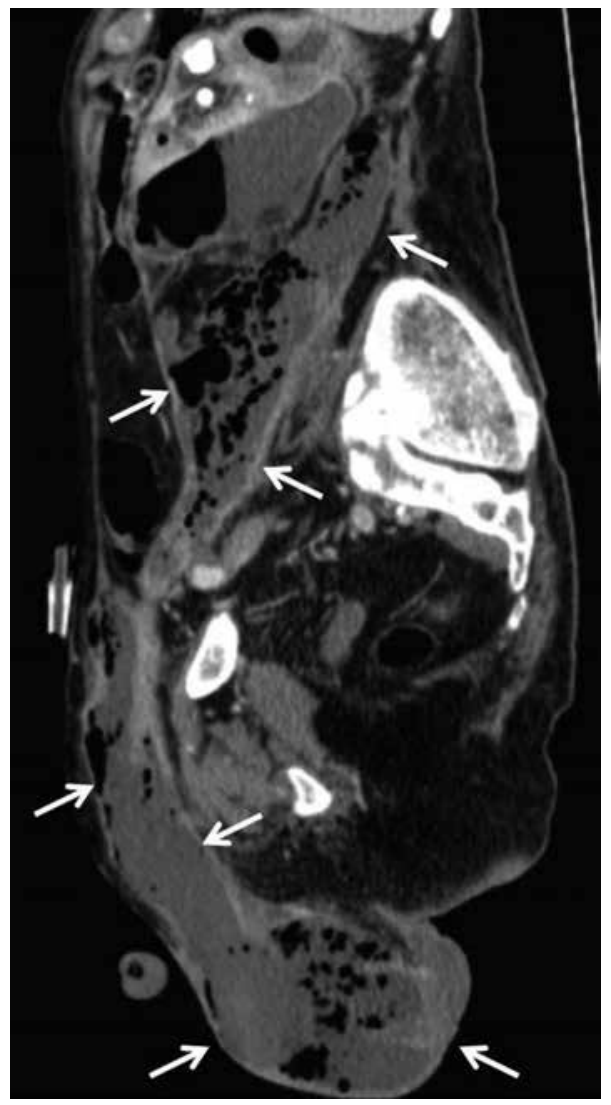

Figure 3. Sagittal computed tomography image of the abdomen shows retroperitoneal extending from retroperitoneal space into the scrotum through the inguinal canal (arrows)

mortality, it is important to consider retroperitoneal abscess in the differential diagnosis and consult radiological examinations without any delay. CT is superior to other methods in the diagnosis and the planning of treatment. It is the most valuable imaging modality to demonstrate anatomical localization and size of the abscess (4).
Direct radiography findings, including abnormal psoas shadow and renal contour, scoliosis, air-fluid levels, ipsilateral hemidiaphragm elevation, basal atelectasis and pleural effusion can be detected in $38-90 \%$ of patients (4). Ultrasonography can be used to follow up the treatment response.

The present case is remarkable in terms of demonstrating that a delayed diagnosis and postponed treatment of a retroperitoneal abscess may lead to spread of the infectious process that may even extend from retroperitoneal space into the scrotum through the inguinal canal and mimic an indirect inguinal hernia.

Concept: Naciye Sinem Gezer

Design: Naciye Sinem Gezer

Data Collection or Processing: Naciye Sinem Gezer, Özkan Alataş, Ali Çaylak, Abdullah Taylan

Analysis or Interpretation: Nuri Karabay, Koray Atila

Literature Search: Özkan Alataş, Ali Çaylak, Abdullah Taylan

Writing: Naciye Sinem Gezer

Peer-review: Internal peer-reviewed.

Conflict of Interest: No conflict of interest was declared by the authors.

Financial Disclosure: The authors declared that this study has received no financial support.

\section{References}

1. Harris LF, Sparks JE. Retroperitoneal abscess. Dig Dis Sci 1980;25:392395.

2. Daviglus GF, Rush BF. Retroperitoneal abscess: a clinical study. Arch Surg 1961;83:322-328.

3. Altemeier WA, Culbertson WR, Fullen WE, Shook CS. Intraabdominal abscesses. Am J Surg 1973;125:70-79.

4. Moody T, Mills P, Cochran T, Williams D. Computerized axial tomography in diagnosis of retroperitoneal abscess. Urology 1980;16:536-538. 